\title{
Determination of meteoroid physical properties from tristatic radar observations
}

\author{
J. Kero ${ }^{1}$, C. Szasz ${ }^{1}$, A. Pellinen-Wannberg ${ }^{1,2}$, G. Wannberg ${ }^{1}$, A. Westman ${ }^{3}$, and D. D. Meisel ${ }^{4}$ \\ ${ }^{1}$ Swedish Institute of Space Physics, Kiruna, Sweden \\ ${ }^{2}$ Umeå University, Kiruna, Sweden \\ ${ }^{3}$ EISCAT Scientific Association, Kiruna, Sweden \\ ${ }^{4}$ SUNY Geneseo, Geneseo, NY, USA
}

Received: 3 January 2008 - Revised: 19 March 2008 - Accepted: 8 April 2008 - Published: 5 August 2008

\begin{abstract}
In this work we give a review of the meteor head echo observations carried out with the tristatic $930 \mathrm{MHz}$ EISCAT UHF radar system during four $24 \mathrm{~h}$ runs between 2002 and 2005 and compare these with earlier observations. A total number of 410 tristatic meteors were observed. We describe a method to determine the position of a compact radar target in the common volume monitored by the three receivers and demonstrate its applicability for meteor studies. The inferred positions of the meteor targets have been utilized to estimate their velocities, decelerations and directions of arrival as well as their radar cross sections with unprecedented accuracy. The velocity distribution of the meteoroids is bimodal with peaks at $35-40 \mathrm{~km} / \mathrm{s}$ and $55-60 \mathrm{~km} / \mathrm{s}$, and ranges from $19-70 \mathrm{~km} / \mathrm{s}$. The estimated masses are between $10^{-9}-10^{-5.5} \mathrm{~kg}$. There are very few detections below $30 \mathrm{~km} / \mathrm{s}$. The observations are clearly biased to high-velocity meteoroids, but not so biased against slow meteoroids as has been presumed from previous tristatic measurements. Finally, we discuss how the radial deceleration observed with a monostatic radar depends on the meteoroid velocity and the angle between the trajectory and the beam. The finite beamwidth leads to underestimated meteoroid masses if radial velocity and deceleration of meteoroids approaching the radar are used as estimates of the true quantities in a momentum equation of motion.
\end{abstract}

Keywords. Interplanetary physics (Interplanetary dust; Instruments and techniques) - Radio science (Instruments and techniques)

\section{Introduction}

Meteor head echoes are radio wave reflections from the plasma generated by the interaction of meteoroids with the

Correspondence to: J. Kero

(kero@irf.se) atmosphere at about $70-140 \mathrm{~km}$ altitude. The echoes are characterized by being transient and Doppler shifted. The received power is confined in range, as from a point source, and the target moves with the line-of-sight velocity of the meteoroid.

The first meteor investigations with what today is termed a High Power Large Aperture (HPLA) radar were conducted by Evans $(1965,1966)$ with the $440 \mathrm{MHz}$ Millstone Hill radar. Some of the measurements were optimized to provide specular trail reflections (Evans, 1965) whereas others were optimized for detecting head echoes of shower meteoroids travelling down-the-beam (Evans, 1966). To study head echoes with a monostatic radar requires that the geometry of the detections be carefully taken into consideration, as only the radial component of the velocity is observed. Evans (1966) pointed the radar beam at shower radiants when these were visible at very low elevations to get as big a crossbeam detection volume as possible and applied strict restrictions to ensure that the detections originated from meteoroids confined in a small angle from boresight. Sporadic meteors do not come from compact radiants and therefore cannot be assumed to be down-the-beam or perpendicular to it.

There was then a 30-year pause in the use of narrow beam HPLA radars for meteor observations, and when they resumed, the improved signal processing techniques and large data handling capacities proved them suitable for studies of sporadic meteor head echoes (Pellinen-Wannberg and Wannberg, 1994; Mathews et al., 1997).

The tristatic capability of the EISCAT UHF system makes it a unique tool in determining meteoroid physical properties and investigations of the head echo scattering process. We present the methods used for meteor head echo observations carried out during four $24 \mathrm{~h}$ runs between 2002 and 2005 as well as a summary of the determined physical characteristics of the observed meteoroids. 
Table 1. Season, year, start/stop dates and times, number of detected tristatic meteors, interpulse period (IPP), pulse repetition frequency (PRF) with two pulses per IPP, pulse length $\left(T_{\text {pulse }}\right)$, and receiver sampling period $\left(T_{\text {sampling }}\right)$ for the meteor campaigns with EISCAT UHF.

\begin{tabular}{|c|c|c|c|c|c|c|c|}
\hline Season & Year & Start-Stop (UT) & No of meteors & $\operatorname{IPP}(\mu \mathrm{s})$ & PRF $(\mathrm{Hz})$ & $T_{\text {pulse }}(\mu \mathrm{s})$ & $T_{\text {sampling }}(\mu \mathrm{s})$ \\
\hline Vernal equinox & 2002 & 19-20 Mar, 12:00-12:00 & 50 & 4334 & 461 & $32 \times 2.0=64.0$ & 0.6 \\
\hline Summer solstice & $\begin{array}{l}2005 \\
2005\end{array}$ & $\begin{array}{l}21-22 \text { Jun, 14:00-10:00 } \\
23 \text { Jun, } 10: 00-14: 00\end{array}$ & 101 & 3312 & 604 & $32 \times 2.4=76.8$ & 0.6 \\
\hline Autumnal equinox & 2005 & 21-22 Sep, 07:00-07:00 & 194 & 3312 & 604 & $32 \times 2.4=76.8$ & 0.6 \\
\hline Winter solstice & 2004 & 21-22 Dec, 08:00-08:00 & 65 & 4334 & 461 & $32 \times 2.4=76.8$ & 0.6 \\
\hline
\end{tabular}

\section{Experiment overview}

The data presented in this study has been collected during four campaigns carried out with the EISCAT UHF radar system as summarized in Table 1. These campaigns were scheduled at vernal/autumnal equinox and summer/winter solstice. A total number of 410 tristatic meteor head echoes contain enough data points for time-of-flight velocity calculations to be compared to the Doppler velocity measurements.

The EISCAT UHF system operates at around $930 \mathrm{MHz}$ and comprises three $32 \mathrm{~m}$ parabolic dish antennae with Cassegrain feeds and $0.6^{\circ}$ one-way half-power beamwidths. One transmitter/receiver is located near Troms $\varnothing$, Norway, and two remote receivers are located in Kiruna, Sweden, and Sodankylä, Finland. The common volume monitored by all three receivers was during all campaigns situated at an altitude of $96 \mathrm{~km}$, about $161 \mathrm{~km}$ in range from Kiruna, $279 \mathrm{~km}$ from Sodankylä and $164 \mathrm{~km}$ from Troms $\varnothing$, as illustrated in Fig. 1. The angle between the Kiruna antenna pointing direction and the Troms $\varnothing$ beam was $75.6^{\circ}$ during the campaigns. The corresponding figure for Sodankylä and Troms $\varnothing$ was $122.2^{\circ}$.

The Doppler frequency measured at a remote receiver is proportional to the sum of the line-of-sight projections of the meteoroid velocity $(v)$ onto the transmitter beam $\left(v_{\mathrm{T}}\right)$ and the receiver beam $\left(v_{R}\right)$ as sketched in Fig. 2. Its magnitude is proportional to the projection of the meteoroid velocity onto the plane spanned by the two beams. The velocity component measured in a bistatic geometry is usually defined as being along the bisector of the transmitter and receiver beams. The meteoroid velocity component along the bisector is calculated by dividing the measured velocity $\left(v_{\mathrm{T}}+v_{\mathrm{R}}\right)$ with $2 \times \cos \gamma$, where $\gamma$ is the bisector angle and equal to the half-angle between the transmitter and the receiver beams.

A 32-bit binary phase shift keyed (BPSK) coded pulse sequence with a bit length of $2.4 \mu \mathrm{s}$ was used in three of the four campaigns (Table 1), giving total pulse lengths of $76.8 \mu$ s (Wannberg et al., 2008). The transmitted waves are left- and the received waves are right-hand circularly polarized.
Three frequencies were used with two pairs of pulse sequences transmitted in each radar cycle, every pair separated by half an interpulse period (IPP). Narrow bandpass filters are used in Sodankylä around $930 \mathrm{MHz}$ to prevent interference from nearby GSM base stations. For this reason, the first pulse sequence in each pair was transmitted at 929.6 MHz in order to be receivable at Sodankylä. To avoid reception of echoes from previously transmitted pulse sequences still within the ionospheric F-layer with the Troms $\varnothing$ transmitter/receiver, the second pulse sequence alternated between 927.5 and $928.7 \mathrm{MHz}$. The whole IPP was $4334 \mu \mathrm{s}$ during the vernal equinox and winter solstice campaigns, which means a separation time of $2167 \mu$ s between consecutive transmissions/receptions. This enables parameters such as meteoroid line-of-sight velocity and echo power to be monitored with a frequency of $461 \mathrm{~Hz}$. The received signals were oversampled by a factor of four at all sites with a $0.6 \mu$ s sampling period. The detection of tristatic pulsating events in the winter solstice data triggered us to shorten the IPP of the later campaigns to $3312 \mu \mathrm{s}$, the lowest achievable IPP with the utilized pulse scheme by reason of beam duty cycle regulations. This gives a monitoring frequency of $604 \mathrm{~Hz}$. The Kiruna reception scheme was also modified to receive both pulses in each pulse pair instead of only one. The two pulses in each pulse pair are separated by $90 \mu \mathrm{s}$.

When decoding the received BPSK coded echo sequences of a meteor echo, the Doppler shift corresponding to the lineof-sight velocity of the meteoroid has to be taken into account. Wannberg et al. (2008) have developed an inverse algorithm in which optimum pulse compression at each individual radar pulse sequence is found by optimizing the Doppler frequency and assumed position of the first meteor echo sample in the dump. In this way, we get two independent measurements of the line-of-sight velocity: a direct estimate of the Doppler velocity, and a very precise estimate of the range rate when comparing the location of meteor echo samples in consecutive dumps.

Previous tristatic EISCAT meteor experiments and results are described by Janches et al. (2002). 


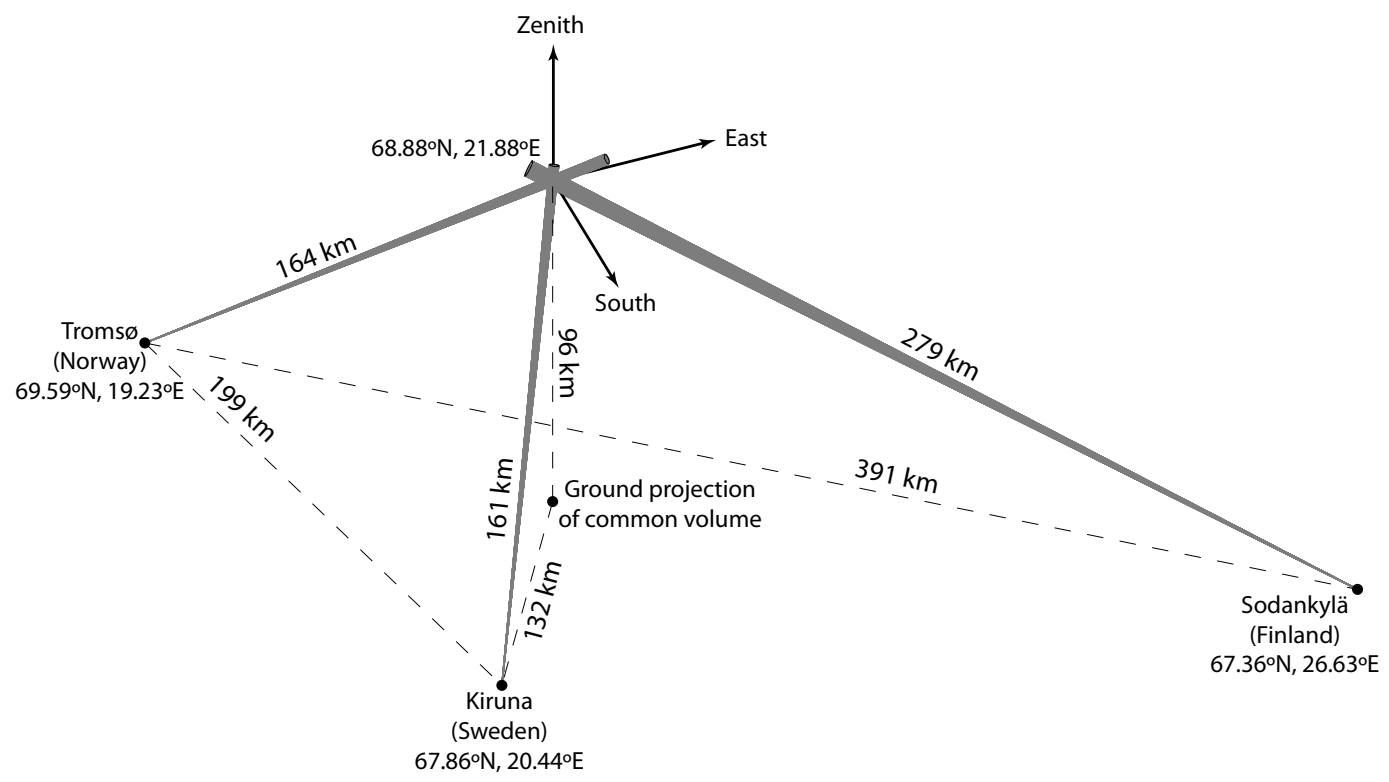

Fig. 1. Sketch of the tetrahedron geometry used in the meteor studies. The full beamwidths are plotted as $1^{\circ}$ and are drawn to scale.

\section{Meteor analysis}

The EISCAT radar system is designed for studying beamfilling, ionospheric plasmas giving rise to incoherent scatter echoes. Hence, compact target position determination has not been realized before. To be able to compare the measured signal-to-noise ratio (SNR) of a head echo streak with the beam pattern, we need to know the amount of transmitted power as well as the antenna receiving sensitivity in the particular direction that a meteor echo arrives from. We have therefore used an ideal radiation pattern (Nygrén, 1996) with a primary reflector size of $30 \mathrm{~m}$, which best matches the measured beamwidth, and a secondary reflector size of $4.48 \mathrm{~m}$ to estimate the antenna gain as a function of an angular displacement from the boresight axis. The maximum gain at boresight is $48 \mathrm{~dB}$. The support structure of the secondary reflector induces azimuthal sidelobe gain variations, which we have not tried to take into account. Therefore we restrict ourselves to the RCS measurements of meteors detected within the $-3 \mathrm{~dB}$ beamwidth.

There are several advantages of knowing the position of a meteor target very precisely. It is not only vital for studying the RCS, but also to get the best possible velocity determination of the targets. When a meteoroid passes through the beam, or rather through the common volume, the angles between the velocity vector and the transmitter and receiver beams change as functions of time. We show in Sect. 5.2 that this geometric effect can not be neglected. In order to estimate the mass and the atmospheric entry velocity of a meteoroid, its deceleration must be determined as precisely as possible. This can only be done if the position of the target

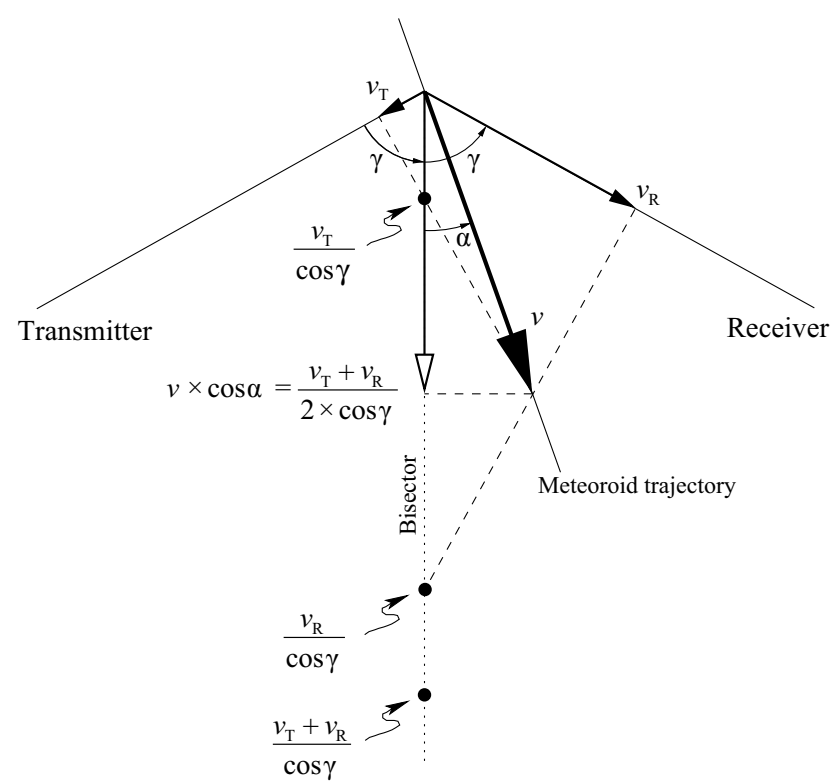

Fig. 2. The Doppler frequency at a remote receiver is proportional to $v_{\mathrm{T}}+v_{\mathrm{R}}$. The velocity component along the bisector is $\left(v_{\mathrm{T}}+v_{\mathrm{R}}\right) /(2 \times \cos \gamma)$.

inside the common volume is known. The deceleration of a meteoroid during the one-tenth of a second it is monitored is usually comparable to, or smaller than, the change in radial velocity of a constant velocity target moving through the beam with the evolving angles not taken into account. 

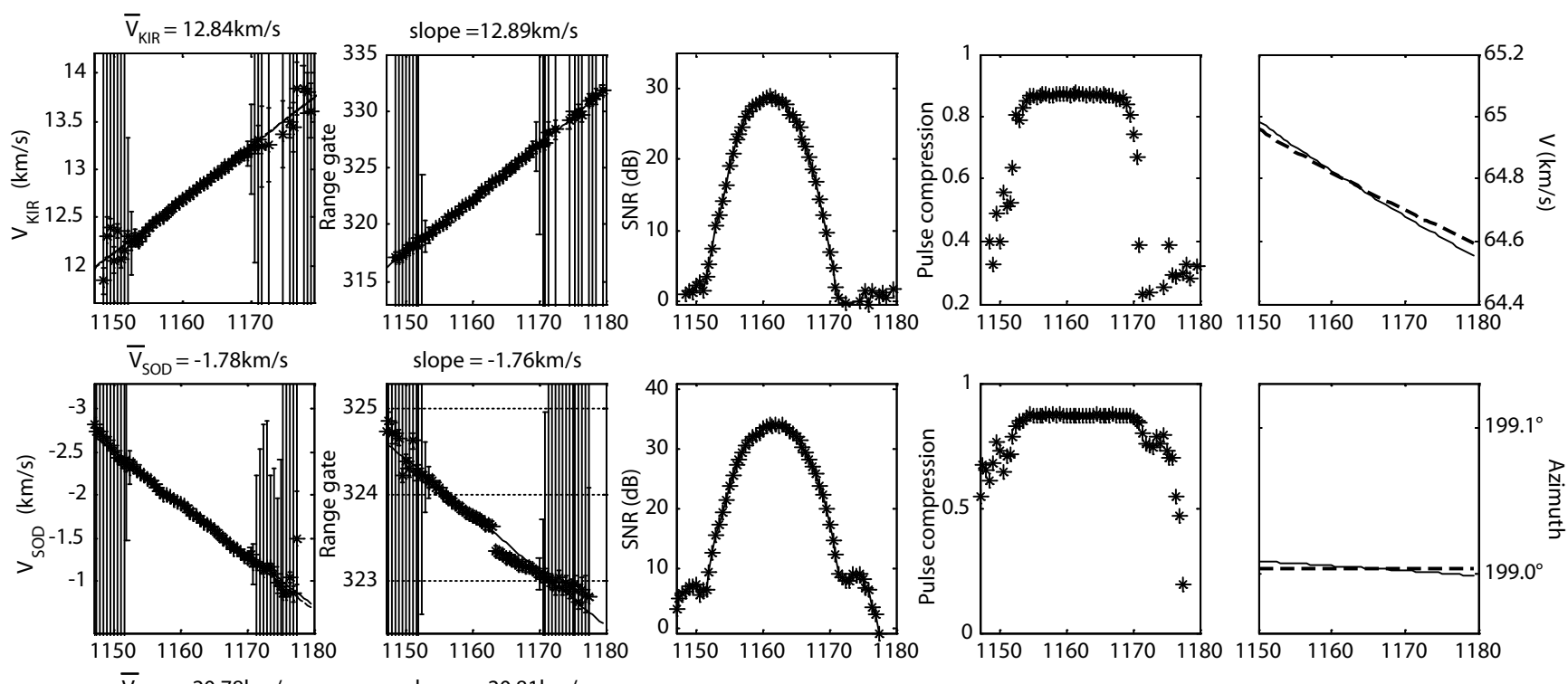

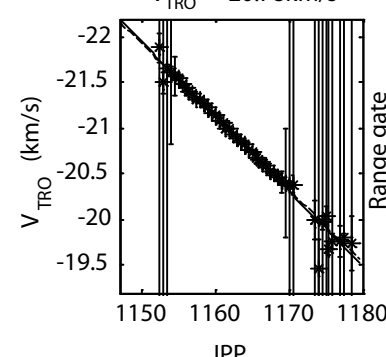

IPP

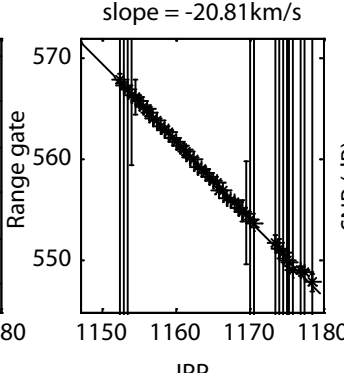

IPP

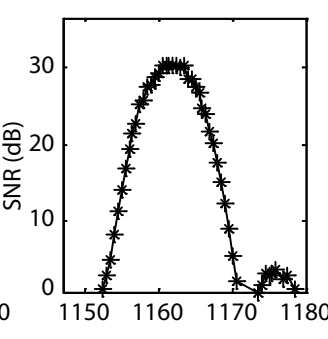

IPP

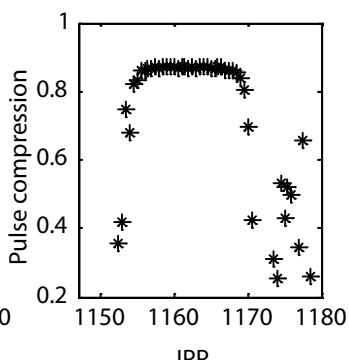

IPP

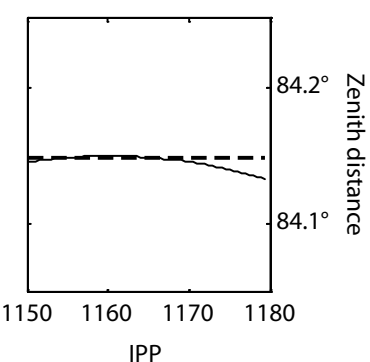

IPP

Fig. 3. Doppler velocity (column 1), range gate (column 2), SNR (column 3), and pulse compression (column 4) versus IPP as seen from Kiruna (row 1), Sodankylä (row 2), and Troms $\emptyset$ (row 3). The rightmost column shows meteoroid velocity (top) azimuth (middle) and zenith distance (bottom) on the ordinates and IPP on the abscissae. The solid lines present the values found when combining the least-squares fitted values of Doppler velocity and range from all receivers. The dashed lines indicate the values found when assuming a straight trajectory. The values given in the subplot headers are the weighted arithmetic mean of the Doppler velocities (column 1) and the slopes of the linear curves fitted to the range data (column 2).

\subsection{Meteor detection}

Figure 3 shows an example of the primary radar parameters deduced for an observed meteor. The top row of the four first columns contains data from Kiruna, the middle row from Sodankylä, and the third row from Troms $\varnothing$. All abscissae are IPP. Each IPP is $3312 \mu$ s long and the whole detection thus lasts for about $0.11 \mathrm{~s}$. The leftmost column shows the Doppler velocity values corresponding to the best frequency found in the decoding process for each receiver. Positive direction is here defined as away from the radar. The second column shows the range gate of the target, the third one displays the SNR, and the fourth one presents the pulse compression. The pulse compression can reach a maximum value of $88 \%$ (Wannberg et al., 2008).

The received signal is oversampled by a factor of four. Hence, each bit in the transmitted code is represented by four points in the received data stream. This is utilized in the fitting procedure to find the position of the peak of the decoded power within a fraction of a range gate with a centre of gravity search routine. The Sodankylä range data (row 2, column 2) demonstrates the accuracy of this method. As the meteoroid trajectory is aligned almost perpendicularly to the Troms $\varnothing$-Sodankylä bisector, the target moves only through two range gates during the observation. Integer values of range are marked by dotted horizontal lines. The estimated target position data points are seen to slightly differ from the weighted least-squares fitted range rate (solid line) in the central part of the panel. There is a gap at IPP 1163, where the range value jumps from 323.63 to 323.35 . The gap occurs when the range to the leading edge of the echo, expressed as an integer value, has shifted by one range gate and is probably best described by a value of about 323.5 . The center-ofgravity search routine seems to be able to find the true target position to an accuracy of at least a third of a range gate, or $30 \mathrm{~m}$. The solid line has a value of 323.55 at the step and a 
slope corresponding to $-1.76 \mathrm{~km} / \mathrm{s}$. The slope is very close to the arithmetic mean of the Doppler velocity $(-1.79 \mathrm{~km} / \mathrm{s})$ and the solid line is estimated to represent the true range of the target to an order of accuracy of one-tenth of a range gate, or $9 \mathrm{~m}$.

The rightmost column in Fig. 3 shows estimated meteoroid velocity (top), as well as the direction of arrival expressed as azimuth (middle) and zenith distance (bottom). The calculations of these quantities are further described in Sect. 3.3.

\subsection{Position determination}

The position of a compact target observed by the three EISCAT UHF receivers can be visualized as situated at the intersection point of three geometrical shapes: a sphere centered around the Troms $\varnothing$ antenna and two prolate spheroidal surfaces, both with Troms $\varnothing$ in one focal point and one of the remote antennae in the other. A prolate spheroidal surface is defined by $x^{2} / a^{2}+\left(y^{2}+z^{2}\right) / b^{2}=1$, where $a$ is the semimajor axis and $b$ is the semi-minor axis. The total range from Troms $\varnothing$ via the target (by definition situated on the surface) to a remote receiver is equal to $2 a$ and can be derived from the measurements. The value of the semi-minor axis $b$ of each spheroid can be readily determined from the identity $b^{2}=a^{2}-c^{2}$, where $2 c$ is the distance between the transmitter and the receiver in the focal points of the spheroid.

The radius of the sphere centred around Troms $\varnothing$ is equal to the monostatic range, $r_{\text {TRO }}$. The position of a target is determined by finding the spatial coordinates $x, y$, and $z$ that simultaneously solve the spheroidal equations of both remote receivers and the spherical equation, $x^{2}+y^{2}+z^{2}=r_{\text {TRO }}^{2}$, with all quantities expressed in a common coordinate system. This has to be performed numerically with an optimization routine as measurement uncertainties are involved.

As it turns out, the measured ranges do not generally provide an accurate position of a target. One first has to determine and correct for some small offsets that are probably caused by group delay in the transmission lines, remote station clock offset, and the small but cumulative errors in the antenna-pointing directions. The last two sources of errors can vary between campaigns even if the experimental setup is identical, but are constant during one measuring occasion.

The total offsets were less than $4 \mu$ s for all experiments, except for an offset of $6.6 \mu \mathrm{s}$ at the 2005 autumnal equinox when the Kiruna GPS receiver had been replaced. The offsets were determined by starting with the assumption that the pointing directions of the antennae were correct. Then we altered the arrival times of the received echoes by small amounts until the set of all trajectories in each experiment came to be grouped around the centre of the common volume. Finally, we plotted the projections of all echoes onto planes perpendicular to each receiver beam to manually check how well the calibration succeeded. In Fig. 4, the pointing direction of the Troms $\emptyset$ beam has been adjusted by

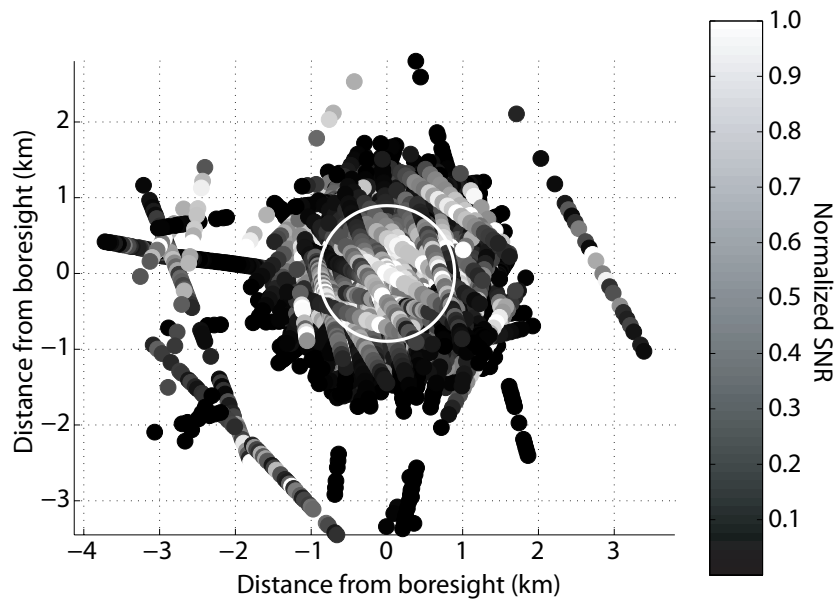

Fig. 4. The 194 meteoroid trajectories observed at autumnal equinox projected onto a plane perpendicular to the Troms $\emptyset$ beam. The maximum SNR of each head echo streak is normalized to one. The white circle marks the $-3 \mathrm{~dB}$ beamwidth $\left(0.6^{\circ}\right)$.

one-tenth of a degree so that its boresight coincides with the concentration point of the head echo streaks. The same is done for Kiruna and Sodankylä independently.

The procedure described above enables us to study the temporal development of the RCS of each and every event in a very efficient way now that we can compensate for the beam pattern. The measured RCS changes very little during many observations, as in the example provided in Fig. 3. The outcome of the position determination algorithm can therefore be verified by comparing the SNR of these events with the beam pattern traced out along the trajectory.

\subsection{Tristatic velocity determination}

To determine the velocity of a meteoroid, we make one assumption and one assumption only: that the meteoroid moves along a straight line through our measurement volume. The accuracy of the range data points is about $30 \mathrm{~m}$ for samples with high SNR. Assuming that there is only one target in the illuminated volume, the range can be found to an even higher precision by making a weighted least-squares fit to the data points. We thus estimate the range to the target from all three antennae at all interpulse periods and calculate the position of the meteoroid from the three ranges as described in Sect. 3.2.

The direction of the meteoroid trajectory is estimated by combining the three measured Doppler velocities (leftmost column in Fig. 3) at the simultaneously derived target positions. The solid lines in the azimuth and zenith distance panels of Fig. 3 (rightmost column) show how these quantities vary when linear least-squares fits of the measured Doppler velocities are combined and fed into a velocity vector calculation routine together with the positions determined using 


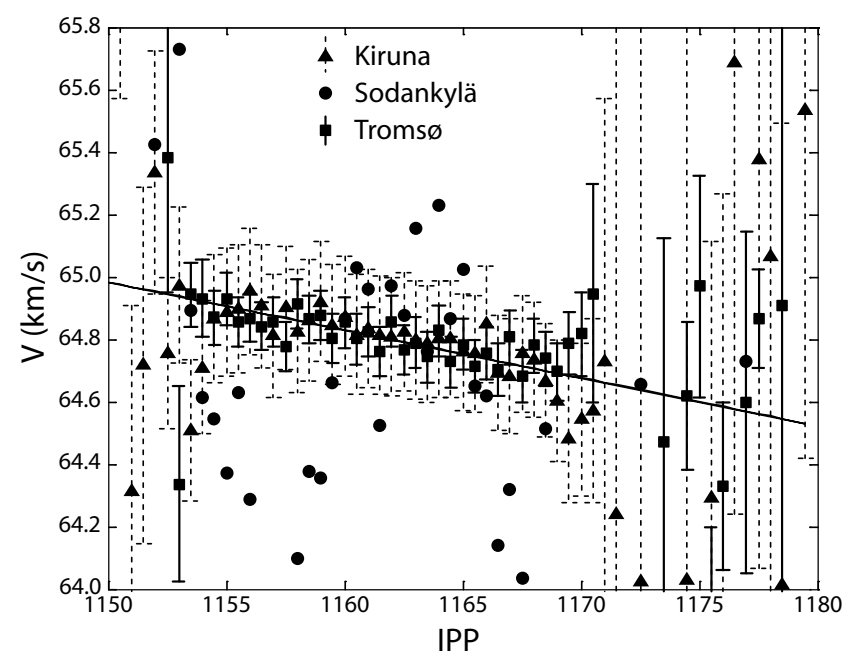

Fig. 5. Velocity estimates from each receiver divided by the cosine of the angle to the derived meteoroid trajectory. Estimated uncertainties for Kiruna and Troms $\varnothing$ are shown as vertical bars. The Sodankylä uncertainties are $\pm 3 \mathrm{~km} / \mathrm{s}$ and are not plotted as they exceed the displayed velocity range.

the linear least-squares fits of the range data. The direction of arrival changes as a function of IPP due to the limited goodness of fit. In this example, the deviation is extremely small. A curvature of a few tenths of a degree is quite common.

The dashed lines in the rightmost column of Fig. 3 represent the direction of arrival calculated from all three receivers at the central IPP of the data set containing the least number of data points, in this case Troms $\emptyset$. Owing to Earth's gravity, a meteoroid follows a curved path. However, the deviation from linearity during the few kilometers of its trajectory within the common volume is small compared to the measurement uncertainties. We therefore assume that the determined direction is the most accurate estimation of the trajectory for the whole duration of the event.

Meteoroid velocity estimates are calculated from each individual Doppler velocity data point. The velocity component values measured at each receiver site are divided by the cosine of the angle from the derived meteoroid trajectory to the direction of the velocity component. Since the angles change with significant amounts when the meteoroid propagates through the measurement volume, they are recalculated using the derived target position at every IPP. The resulting meteoroid velocity estimates are plotted in Fig. 5. An error in the derived direction of the trajectory would reveal itself as a systematic difference between the calculated meteoroid velocity estimates from each site. The length of the vertical bars plotted for the Kiruna and Troms $\emptyset$ data in Fig. 5 show the estimated uncertainties. The Sodankylä data uncertainties are of the order of $\pm 3 \mathrm{~km} / \mathrm{s}$ and are not shown in the graph as they exceed the displayed velocity range.
The uncertainty of a meteoroid velocity data point is estimated as inversely proportional to the pulse compression squared, divided by the cosine of the angle between the measured velocity component and the determined trajectory. The uncertainty in the Troms $\varnothing$ velocity data for this event is in case of high pulse compression estimated to $\pm 0.08 \mathrm{~km} / \mathrm{s}$ (solid bars). The uncertainty in the data from Kiruna is estimated to $\pm 0.2 \mathrm{~km} / \mathrm{s}$ (dashed bars), larger than the uncertainty in the Troms $\varnothing$ data due to the greater angle from the Kiruna bisector to the trajectory than from the velocity component measured in Troms $\varnothing$. The scatter of the Sodankylä data is caused by its bisector and the trajectory being almost perpendicular.

Weights used for determining least-squares fits to the velocity data are chosen as inversely proportional to the estimated uncertainties. Hence, both the pulse compressions and the geometry of the trajectory are used to calculate them.

The accuracy of the meteoroid velocity determination is better when using this method compared to combining the simultaneously estimated velocity components from all three receivers to calculate both speed and direction at each IPP. There is another important advantage as well: several events have low SNR and provide only a few data points from one or two of the receivers, but good SNR and a long series of measurements from the other/-s. A few data points from each receiver are enough for an accurate direction determination. If at least one of the receivers provides a long sequence of data, the deceleration can also be deduced.

The use of the position of the target at each IPP cancels the effect of the changing angle as the meteoroid traverses the measurement volume. This is important for accurate velocity calculation and thus crucial for deceleration determination.

The slanted solid line in Fig. 5 is a weighted linear leastsquares fit to the calculated meteoroid velocity estimates. In general, we do not try to describe the velocity with a polynomial or exponential function, but fit the data directly to an ablation model to estimate the meteoroid mass and the atmospheric entry velocity (Szasz et al., 2007).

The ratios between the meteoroid deceleration in Fig. 5 and the radial acceleration/deceleration evident in the Kiruna, Sodankylä and Troms $\varnothing$ data (Fig. 3, column 1) are clearly different from the ratios between the meteoroid velocity and the radial velocities. This behaviour is due to the effect of finite beamwidth on radial velocity measurements and is further discussed in Sect. 5.2. The radial velocity of a moving target is biased by its transversal velocity component.

\subsection{Meteoroid mass estimation}

A standard way of estimating the mass of a meteoroid is to use its determined velocity and deceleration in a momentum equation (Bronshten, 1983). Travelling through the atmosphere, a meteoroid decelerates due to collisions with the atmospheric constituents. To also take into account that 


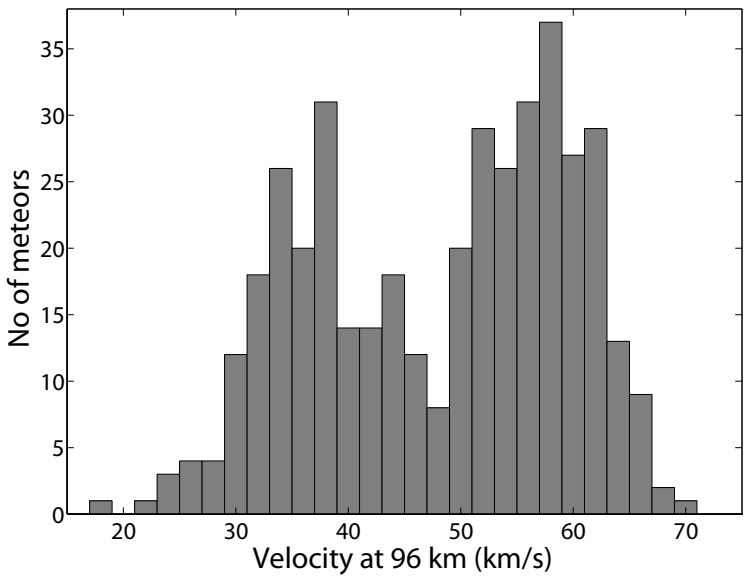

Fig. 6. Distribution of observed velocities.

the mass of a meteoroid changes due to ablation during an observation, we have implemented the standard meteoroidatmosphere interaction processes (Öpik, 1958) in a numerical single-object ablation model to compare with our observations. Ablation is the collective term for several kinds of mass loss including vaporization, sublimation, fusion and loss of molten droplets. The model is implemented in a way similar to that of Rogers et al. (2005) and references therein, originally based on Öpik (1958), Bronshten (1983) and Love and Brownlee (1991), with the addition of a sputtering model as described by Tielens et al. (1994). The input meteoroid parameters to the model are above-atmosphere velocity, mass, density and zenith distance. MSIS-E-90 (Hedin, 1991) is used for atmospheric densities. The measured RCS is assumed to be proportional to the meteoroid mass loss via an overdense scattering mechanism (Close et al., 2002; Westman et al., 2004). The model is further described by Szasz et al. (2007) and in detail by Kero (2008).

The velocity and RCS data of each meteor is compared and fitted to the ablation model by adjusting the input parameters propagated down through the atmosphere to our observation altitude using a fifth order Runge-Kutta numerical integration technique with a variable step size (Danby, 1988). Here we focus on the tristatic radar measurement technique and suffice it to say that the data could be employed together with any kind of physical model describing the atmosphere interaction processes, e.g., the ablation model described by Campbell-Brown and Koschny (2004), the analytical scattering model by Close et al. (2004), or the numerical simulations by Dyrud et al. (2007).

\section{Results}

The velocity distribution of the detected meteors is reported in Fig. 6. None of the meteoroids has a speed higher than

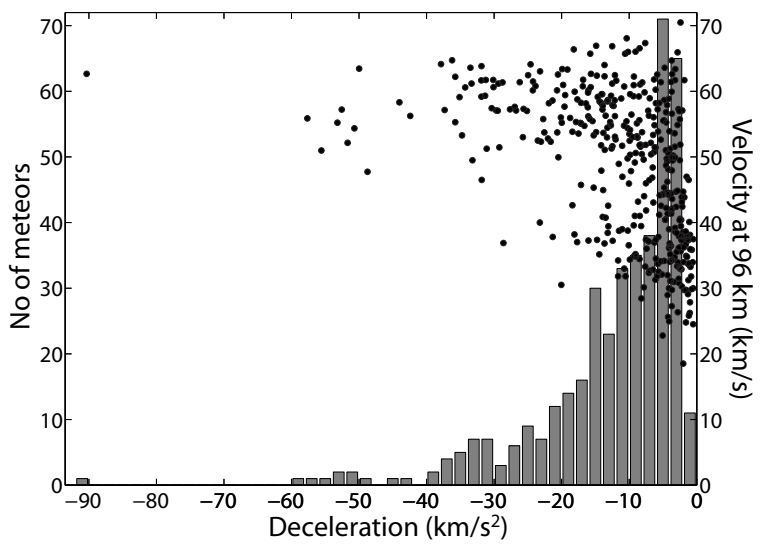

Fig. 7. Distribution of observed deceleration and a scatter plot of the velocity versus deceleration.

$72 \mathrm{~km} / \mathrm{s}$, which is the highest geocentric speed of an object on a bound orbit in the solar system. The distribution is bimodal with peaks at $35-40 \mathrm{~km} / \mathrm{s}$ and $55-60 \mathrm{~km} / \mathrm{s}$. From the measurements, Szasz et al. (2008) have estimated the atmospheric entry velocities and calculated the heliocentric orbits of the meteoroids.

The deceleration distribution is presented as a histogram as well as a scatter plot showing velocity versus deceleration in Fig. 7. The displayed decelerations are estimated linearly to enable a comparison with other reported values. It is evident from the scatter plot that high deceleration implies high velocity, but not necessarily vice versa. This trend is probably due to faster meteoroids being at the end of their trajectories and losing mass more rapidly at the altitude of the common volume than slow meteoroids on similar trajectories. Only a few $\mathrm{km}$ of each meteoroid trajectory is observed. The measured deceleration must therefore not be confused with the total deceleration.

The RCS distribution of the 265 tristatic meteors that appear inside the $-3 \mathrm{~dB}$ width of the Troms $\varnothing$ beam is provided in Fig. 8 as a histogram and a scatter plot of observed velocity versus RCS. Kero et al. (2008b) have shown that the RCSs measured simultaneously at the three receivers are equivalent and that the targets have similar properties at aspect angles all the way out to $130^{\circ}$ from the direction of propagation of a meteoroid. This suggests that the head echo target is essentially spherical in the forward direction, consistent with the polarization measurements by Close et al. (2002) and the plasma and electromagnetic simulations of meteor head echoes performed by Dyrud et al. (2007).

A histogram of the meteoroid masses estimated at the starting altitude of each observation and a scatter plot of velocity versus mass is displayed in Fig. 9. The masses range from $10^{-9.5}$ to $10^{-6} \mathrm{~kg}$. The distribution of the data in the scatter plot agrees with the general notion that slow, lowmass meteoroids escape detection with HPLA radars (Close 


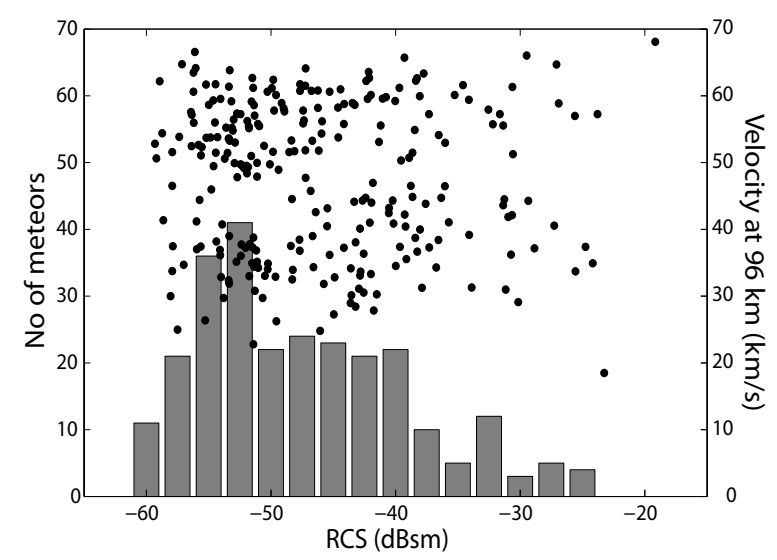

Fig. 8. Distribution of the monostatic RCS measured in Troms $\varnothing$ for the 265 meteors appearing inside the $-3 \mathrm{~dB}$ beamwidth and a scatter plot of the velocity versus RCS. The RCS is expressed as dB relative to a square metre $(\mathrm{dBsm})$.

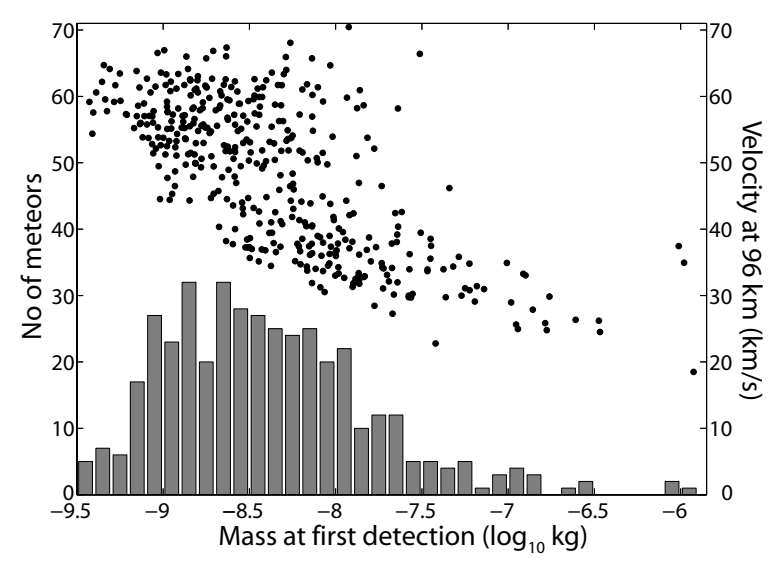

Fig. 9. Distribution of estimated meteoroid masses at detection and a scatter plot of velocity versus mass.

et al., 2007), but we cannot draw any firm observational conclusion on the correlation between velocity and mass as the masses are affected by ablation model assumptions. In the momentum equation of the ablation model, the velocity $(v)$ to deceleration $(\dot{v})$ ratio is proportional to mass $(m)$ via a power law (Bronshten, 1983), where $m \propto v^{6} / \dot{v}^{3}$.

Figure 10 presents the distribution of the modelled atmospheric entry masses, which is very similar to the mass distribution found by Close et al. (2007) for the meteors detected with ALTAIR (Advanced Research Projects Agency LongRange Tracking and Instrumentation Radar). Since we use a single-body ablation model, the estimated masses are not truly representative for fragmenting meteoroids. Fragmentation will always increase the deceleration, but often increase the RCS as well. These two effects in combination are similar in the ablation model to the choice of a lower meteoroid

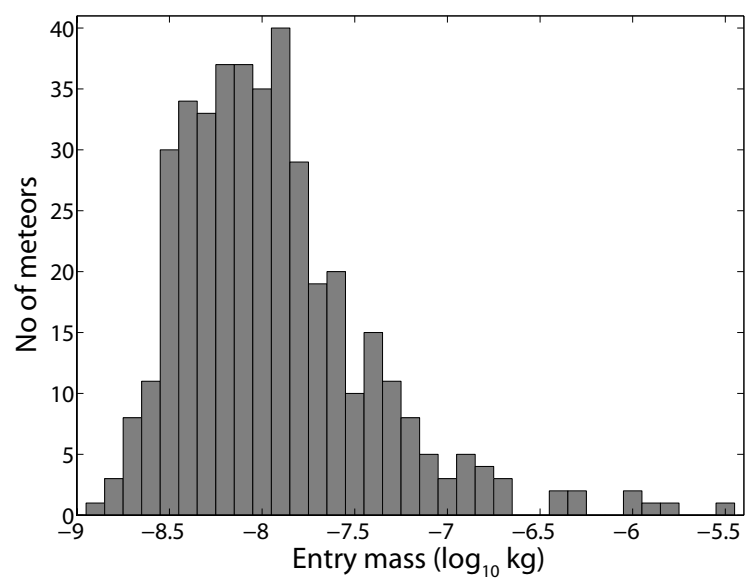

Fig. 10. Distribution of estimated atmospheric entry masses for the detected meteoroids.

density. This results in a larger particle cross-sectional area over mass ratio, gives an enhanced mass loss for the same meteoroid mass and increases both the RCS and the deceleration as compared to a particle with a higher density. The mass determined for a fragmenting meteoroid is therefore an underestimation of the total mass of the fragments.

Szasz et al. (2007) have modelled the luminosity of the detected meteors and estimate them to be in the range of +9 to +5 absolute visual magnitude.

\section{Discussion}

\subsection{Comparisons with previous results}

The velocity distribution displayed in Fig. 6 differs from previous EISCAT measurements. Eight out of ten observed tristatic EISCAT UHF meteors analyzed by Janches et al. (2002) were determined to have velocities higher than $60 \mathrm{~km} / \mathrm{s}$, the fastest one estimated to $86 \mathrm{~km} / \mathrm{s}$. It has therefore been presumed that the radar detections are heavily biased to high-velocity meteoroids. The RCS distribution of the present observations reported in Fig. 8 shows that there are approximately as many large targets in the slower part of the bimodal velocity distribution as there are in the faster part. On the other hand, the present velocity distribution also exhibits a drop in the number of detections below about $30 \mathrm{~km} / \mathrm{s}$ and is still clearly biased to high-velocity meteoroids.

\subsection{Radial deceleration due to finite beamwidth}

The phase fronts in the far-field region of a transmitter antenna are not planer, but spherical. The Doppler shift of the transmitted wave incident on a compact target moving along a straight trajectory with an angle to the beam depends therefore on where in the beam the target is located. A 
simple sketch is provided in Fig. 11. Similarly, the scattered, or reflected, wave from a compact target generates spherical phase fronts at the receiver. Only the part of a spherical phase front for which the phase is inside one Fresnel zone contributes to the signal at the antenna output. Wave propagation satisfies reciprocity. The Doppler shift of the received signal at the antenna output is therefore proportional to $2 \times v \times \cos \alpha$, where $v$ is the target velocity and $\alpha$ is the angle between a ray from the center of the antenna to the target.

The effect of beamwidth is very obvious when a meteoroid is receding from the radar. The meteoroid in the example displayed in Fig. 3 is crossing the Kiruna bisector from behind. Hence the angle between the trajectory and the bisector decreases with time. The Kiruna Doppler velocity therefore increases at $+16.8 \mathrm{~km} / \mathrm{s}^{2}$ (from $\simeq 12 \mathrm{~km} / \mathrm{s}$ to $\left.\simeq 13.5 \mathrm{~km} / \mathrm{s}\right)$. At the same time, the Sodankylä Doppler velocity is decreasing at $-18.0 \mathrm{~km} / \mathrm{s}^{2}$, and the Troms $\varnothing$ velocity at $-24.8 \mathrm{~km} / \mathrm{s}^{2}$. The meteoroid is approaching both of these receivers. The radial velocity gradients are almost an order of magnitude larger than the true deceleration plotted in Fig. 5 and estimated to $-3.8 \mathrm{~km} / \mathrm{s}^{2}$.

Figure 12 shows the radial deceleration as a function of meteoroid velocity and aspect angle for a non-decelerating meteoroid and a meteoroid decelerating at $-20 \mathrm{~km} / \mathrm{s}^{2}$. In both cases, the target is assumed to approach a monostatic radar at a range of $100 \mathrm{~km}$. We have calculated the radial deceleration by letting the beamwidth approach zero. The radial velocity changes non-linearly as a function of time during the passage through a wide beam. The values given in Fig. 12 are equal to the straight line tangents at the boresight axis.

It is evident from Fig. 12a that the radial deceleration of a constant velocity meteoroid increases both as a function of meteoroid velocity and aspect angle. The radial acceleration of a receding meteoroid has the same magnitude as the radial deceleration of a meteoroid approaching from the opposite direction. If the aspect angle is closer than the beamwidth to $90^{\circ}$, the Doppler velocity switches sign during the observation.

The radial deceleration dependence on velocity and aspect angle of a decelerating meteoroid is not as intuitive as in the constant velocity example. For a target decelerating at $-20 \mathrm{~km} / \mathrm{s}^{2}$, as displayed in Fig. 12b, the radial deceleration decreases with aspect angle if the velocity is below about $30 \mathrm{~km} / \mathrm{s}$, but increases if it travels faster than that.

\subsection{Comparisons with other high-latitude HPLA radars}

Mathews et al. (2007) have compared measurements from the $430 \mathrm{MHz}$ Arecibo Observatory UHF radar (AO), the $1290 \mathrm{MHz}$ Søndre Strømfjord Research Facility (SRF) and the 449.3 MHz 32 panel Poker Flat Advanced Modular Incoherent Scatter Radar (PF AMISR-32). These radars have all been used in monostatic mode with a vertical or almost vertical pointing direction. The radial decelerations can therefore

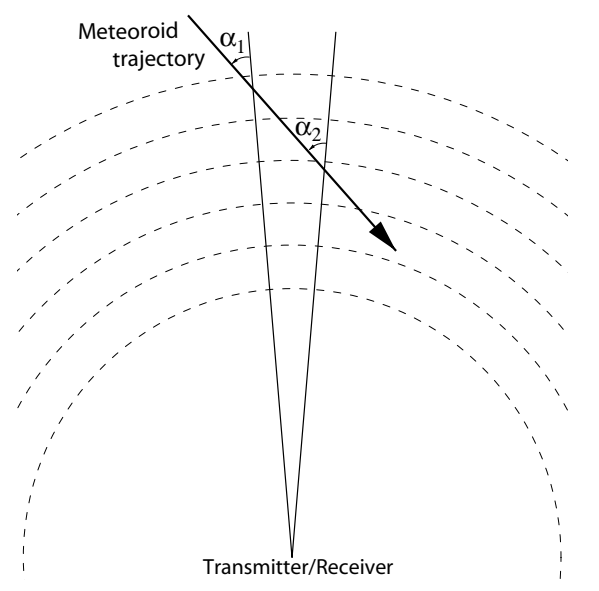

Fig. 11. Sketch of a meteoroid trajectory in the far-field of a radar beam (solid lines), where the phase fronts are spherical (dashed lines). The Doppler shift varies as $\cos \alpha$ along the trajectory. For an approaching meteoroid: $\alpha_{1}<\alpha_{2}$.

be as much as $55 \mathrm{~km} / \mathrm{s}^{2}$ larger than the true decelerations, as illustrated in Fig. 12. This is, however, negligible compared to the largest reported values of deceleration that are greater than $1000 \mathrm{~km} / \mathrm{s}^{2}$. The highest values of decelerations are observed with SRF and PF AMISR-32. Both these facilities are located near the Arctic Circle at latitudes comparable with the EISCAT UHF system.

To compare the facilities, Mathews et al. (2007) use a quality factor given by transmitted power, $P_{T}$, the effective aperture, $A_{\text {eff }}$, and the system temperature, $T_{\text {sys. }}$. The so-defined quality factor has a value of about

$\frac{P_{T} \times A_{\text {eff }}}{T_{\text {sys }}} \simeq \frac{1.3 \mathrm{MW} \times 800 \mathrm{~m}^{2}}{115 \mathrm{~K}} \simeq 9 \mathrm{MW} \mathrm{m}^{2} / \mathrm{K}$

for the EISCAT UHF system while AO, SRF and PF AMISR-32 have $1.5 \times 10^{3}, 19$, and $0.67 \mathrm{MWm}^{2} / \mathrm{K}$, respectively (Mathews et al., 2007). Being so big, AO is in a class of its own. Yet events with large decelerations are detected with SRF and PF AMISR-32 as well. Since the EISCAT UHF has both a quality factor and an operating frequency (at $930 \mathrm{MHz}$ ) between those of these two radars, it should also observe these events.

The rate of events recorded during morning hours (04:0008:00 LT) at the end of July and beginning of August was about $34 \mathrm{~h}^{-1}$ for SRF and $55 \mathrm{~h}^{-1}$ for PF AMISR-32 (Mathews et al., 2007). The rate of monostatic detections within a similar altitude interval during the same time of day with EISCAT Troms $\varnothing$ UHF was $30-40 \mathrm{~h}^{-1}$ at summer solstice and $50-60 \mathrm{~h}^{-1}$ at autumnal equinox. These rates are comparable to those from SRF and PF AMISR-32, but the tristatic meteor rates are limited to the extent of the common volume of all three receivers, an altitude interval of about $96 \pm 2 \mathrm{~km}$, and are therefore much lower $\left(10-20 \mathrm{~h}^{-1}\right)$. The deceleration 
(a)

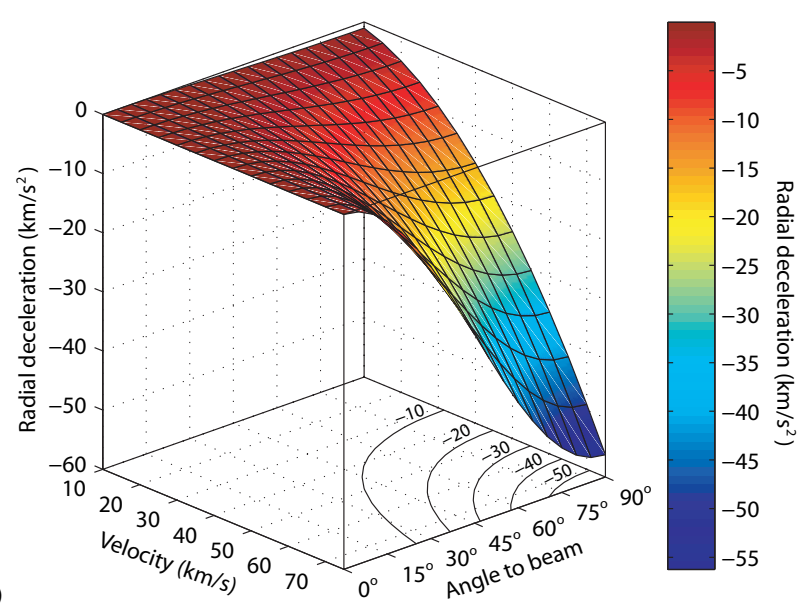

(b)

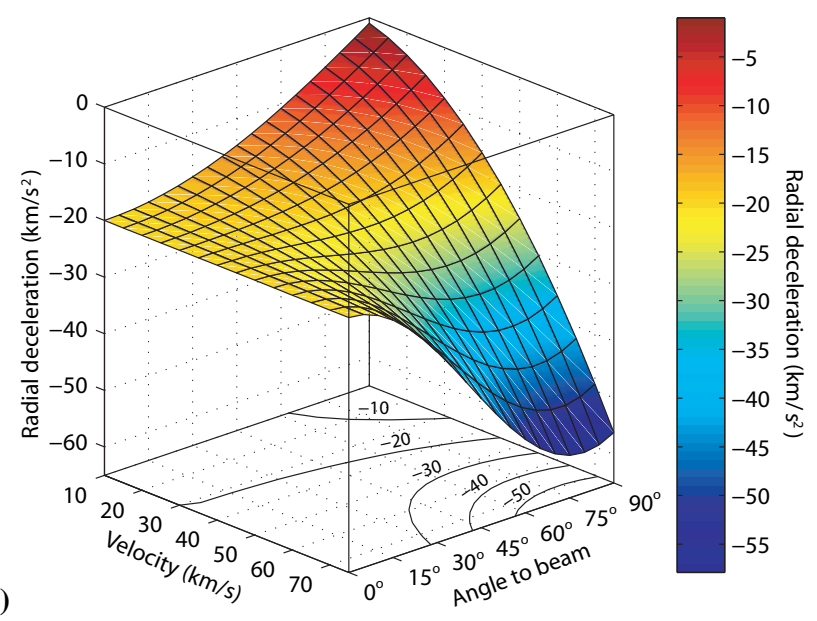

Fig. 12. Radial deceleration as a function of meteoroid velocity and angle from the trajectory to the beam (aspect angle) for (a) a nondecelerating meteoroid and (b) a meteoroid decelerating at $-20 \mathrm{~km} / \mathrm{s}^{2}$ in the far-field at a range of $100 \mathrm{~km}$ from a radar. The radial deceleration is estimated as a linear value by letting the beamwidth approach zero. The contours in the bottom of the charts are projected isolines of radial deceleration.

distribution of the tristatic meteors given in Fig. 7 has a much smaller spread than the distributions reported by Mathews et al. (2007). The total number of detected meteors was 271 with SRF and 443 with PF AMISR-32. The events with high deceleration are scattered over a large altitude interval, which includes $96 \mathrm{~km}$.

From the present analysis we conclude that the EISCAT UHF data contain no meteoroids with very large decelerations. If they are not artifacts of the data analysis methods applied by Mathews et al. (2007), their absence in our observations means we have to use a different approach: to find large decelerations we may have to investigate the few data points in the very end of each event more carefully. Alternatively, the initial quality control must be altered where events are excluded if the time-of-flight velocity cannot be compared with the Doppler velocity. It might be necessary to accept a smaller number of Doppler velocity data points and higher uncertainty than in the present method of analysis. This will be investigated in a future study. A meteoroid of large enough mass to produce detectable ionization must break into small dust grains to decelerate this fast since a very high momentum transfer is required. The individual grains of a disrupted meteoroid have high cross-section area over mass ratio and can together provide a transient amount of detectable ionization. We do not expect to be able to detect any ionization produced along the atmospheric trajectory of one single micron-sized particle.

\subsection{Factors that may affect the determined velocity}

McKinley and Millman (1949) were the first to suggest that the meteor head echo targets are compact regions of plasma, co-moving with the meteoroids, and virtually independent of aspect. We find no reason to assume otherwise and use the determined target velocities as representative for the meteoroids themselves. There are, however, a few implications that may introduce differences between the observed velocity and the meteoroid velocity.

Some meteor observations contain regular pulsations in the received power. Kero et al. (2008a) have shown that these are sometimes caused by interference between echoes from two or more distinct scattering centers simultaneously present in the radar beam. The Doppler shift of the returned signal from two simultaneously illuminated meteoric fragments is proportional to a weighted arithmetic mean of their Doppler velocities, with weights equal to the square root of their RCSs. The pulsation rates are often seen to increase, a behaviour consistent with two fragments of unequal crosssectional area over mass ratio separating from each other due to different decelerations along the trajectory of their parent meteoroid. The pulsation rate at a remote receiver is proportional to the sum of the projections of their differential velocity onto the transmitter and receiver beams in analogy with the Doppler shift of the transmitted frequency. The component of the differential velocity along the bisector is found by dividing the detected pulsation rate with $2 \times \cos \gamma$ (as defined in Fig. 2). 
Close (2004) indicates another reason why there may be a difference between the observed range rate of a meteor target and the meteoroid velocity. If the radar target associated with a meteoroid scales as the atmospheric mean-free path, there may be a significant time delay between the passage of the meteoroid and the formation of the radar target at high altitudes. Owing to the exponentially increasing atmospheric density and consequently shorter mean-free path of ablated atoms, the time delay decreases as the meteoroid penetrates deeper into the atmosphere. Close et al. (2004) argues that this effect in practice could give an acceleration term that must be subtracted from the observed velocity. This would explain some anomalous ALTAIR observations where the head echo targets seem to accelerate.

The tristatic measurements with the EISCAT UHF radar extend over a rather limited altitude interval around $96 \mathrm{~km}$ and do not contain any accelerating events.

\section{Conclusions}

The tristatic EISCAT UHF system has been used in a series of measurement campaigns from 2002-2005 to study meteoric head echoes. 410 meteors were simultaneously detected with all three receivers and contained enough data points for time-of-flight velocity calculations to be compared with the Doppler velocity measurements. We have presented the meteor analysis methods used and how velocity, deceleration, RCS and meteoroid mass are estimated from the observations.

The velocities of the meteoroids giving rise to the head echoes are scattered from 19 to $70 \mathrm{~km} / \mathrm{s}$ in a bimodal distribution, but with very few detections below $30 \mathrm{~km} / \mathrm{s}$. The estimated masses are in the range $10^{-9}-10^{-5.5} \mathrm{~kg}$ and distributed very similarly to the masses determined for the ALTAIR head echo detections by Close et al. (2007).

Many monostatic radar observations are conducted with a vertically pointed beam. Meteoroids will always approach the radar with this measurement geometry. We have demonstrated that the effect of finite beamwidth leads to a radial deceleration that is larger than the true deceleration $(\dot{v})$ for an approaching meteoroid. The radial velocity is, however, always smaller than the true velocity $(v)$. The use of radial deceleration and radial velocity in a momentum equation where $m \propto v^{6} / \dot{v}^{3}$ (Bronshten, 1983) therefore gives an underestimated meteoroid mass $(m)$.

Acknowledgements. We gratefully acknowledge the EISCAT staff for their assistance during the experiment. EISCAT is an international association supported by research organizations in China (CRIPR), Finland (SA), France (CNRS), Germany (DFG), Japan (NIPR and STEL), Norway (NFR), Sweden (VR) and the United Kingdom (STFC). Two of the authors (JK and CS) are supported by the Swedish National Graduate School of Space Technology. We thank M. Campbell-Brown for valuable comments which have improved this paper.
Topical Editor K. Kauristie thanks M. Campbell-Brown and L. Dyrud for their help in evaluating this paper.

\section{References}

Bronshten, V. A.: Physics of Meteoric Phenomena, D. Reidel Publishing Company, 1983.

Campbell-Brown, M. D. and Koschny, D.: Model of the ablation of faint meteors, A\&A, 418, 751-758, doi:10.1051/0004-6361: 20041001, 2004.

Close, S.: Theory and Analysis of Meteor Head Echoes and Meteoroids using High-Resolution Multi-Frequency Radar Data, Ph.D. thesis, Boston University, 2004.

Close, S., Oppenheim, M., Hunt, S., and Dyrud, L.: Scattering characteristics of high-resolution meteor head echoes detected at multiple frequencies, J. Geophys. Res. (Space Physics), 107, 9-1, doi:10.1029/2002JA009253, 2002.

Close, S., Oppenheim, M., Hunt, S., and Coster, A.: A technique for calculating meteor plasma density and meteoroid mass from radar head echo scattering, Icarus, 168, 43-52, doi:10.1016/j. icarus.2003.11.018, 2004.

Close, S., Brown, P., Campbell-Brown, M., Oppenheim, M., and Colestock, P.: Meteor head echo radar data: Mass velocity selection effects, Icarus, 186, 547-556, doi:10.1016/j.icarus.2006.09. 007, 2007.

Danby, J. M. A.: Fundamentals of celestial mechanics, WillmannBell, 2nd. rev. and enlarged edn., 1988.

Dyrud, L., Wilson, D., Boerve, S., Trulsen, J., Pecseli, H., Close, S., Chen, C., and Lee, Y.: Plasma and electromagnetic wave simulations of meteors, Adv. Space Res., 102, 383-394, doi:10.1016/j.asr.2007.03.048, 2007.

Evans, J. V.: Radio-echo studies of meteors at 68-centimeter wavelength, JGR, 70, 5395-5416, 1965.

Evans, J. V.: Radar Observations of Meteor Deceleration, JGR, 71, 171-188, 1966.

Hedin, A. E.: Extension of the MSIS thermosphere model into the middle and lower atmosphere, J. Geophys. Res., 96, 1159-1172, 1991.

Janches, D., Pellinen-Wannberg, A., Wannberg, G., Westman, A., Häggström, I., and Meisel, D. D.: Tristatic observations of meteors using the $930 \mathrm{MHz}$ European Incoherent Scatter radar system, J. Geophys. Res., 107, 14-1, 2002.

Kero, J.: High-resolution meteor exploration with tristatic radar methods, Ph.D. thesis, Swedish Institute of Space Physics, Kiruna, Sweden, 2008.

Kero, J., Szasz, C., Pellinen-Wannberg, A., Wannberg, G., Westman, A., and Meisel, D. D.: Three dimensional radar observation of a submillimeter meteoroid fragmentation, Geophys. Res. Lett., 35, L04 101, doi:10.1029/2007GL032733, 2008a.

Kero, J., Szasz, C., Wannberg, G., Pellinen-Wannberg, A., and Westman, A.: On the meteoric head echo radar cross section angular dependence, Geophys. Res. Lett., 35, L07101, doi:10.1029/2008GL033402, 2008b.

Love, S. G. and Brownlee, D. E.: Heating and thermal transformation of micrometeoroids entering the Earth's atmosphere, Icarus, 89, 26-43, 1991.

Mathews, J. D., Meisel, D. D., Hunter, K. P., Getman, V. S., and Zhou, Q.: Very High Resolution Studies of Micrometeors Using 
the Arecibo $430 \mathrm{MHz}$ Radar, Icarus, 126, 157-169, doi:10.1006/ icar.1996.5641, 1997.

Mathews, J. D., Briczinski, S. J., Meisel, D. D., and Heinselman, C. J.: Radio and meteor science outcomes from comparisons of meteor radar observations at AMISR Poker Flat, Sondrestrom and Arecibo, EM\&P, 102, 365-372, doi:10.1007/s11038-0079168-0, 2007.

McKinley, D. W. R. and Millman, P. M.: A phenomenological theory of radar echoes from meteors, Proc. IRE, 37, 364-375, 1949.

Nygrén, T.: Introduction to incoherent scatter measurements, Invers Oy, 1st edn., 1996.

Öpik, E. J.: Physics of meteor flight in the atmosphere, no. 6 in Interscience tracts on physics and astronomy, Interscience Publishers, Inc., 1958.

Pellinen-Wannberg, A. and Wannberg, G.: Meteor observations with the European Incoherent Scatter UHF radar, JGR, 99, $11379-11390,1994$.

Rogers, L. A., Hill, K. A., and Hawkes, R. L.: Mass loss due to sputtering and thermal processes in meteoroid ablation, Planet. Space Sci, 53, 1341-1354, doi:10.1016/j.pss.2005.07.002, 2005.
Szasz, C., Kero, J., Pellinen-Wannberg, A., Meisel, D. D., Wannberg, G., and Westman, A.: Estimated visual magnitudes of the EISCAT UHF meteors, EM\&P, 102, 373-378, doi:10.1007/s11038-007-9206-y, 2007.

Szasz, G., Kero, J., Meisel, D. D., Pellinen-Wannberg, A., Wannberg, G., and Westman, A.: Orbit characteristics of the tristatic EISCAT UHF meteors, MNRAS, in press, 2008.

Tielens, A. G. G. M., McKee, C. F., Seab, C. G., and Hollenbach, D. J.: The physics of grain-grain collisions and gas-grain sputtering in interstellar shocks, Astrophys. J., 431, 321-340, doi: 10.1086/174488, 1994.

Westman, A., Wannberg, G., and Pellinen-Wannberg, A.: Meteor head echo altitude distributions and the height cutoff effect studied with the EISCAT HPLA UHF and VHF radars, Ann. Geophys., 22, 1575-1584, 2004, http://www.ann-geophys.net/22/1575/2004/. 